\title{
Artículo
}

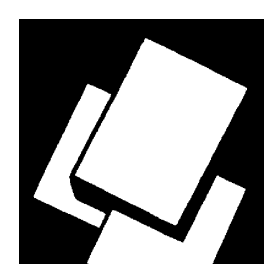

\section{La prejubilación en los miembros de alta dirección de las organizaciones: un análisis cualitativo}

\section{Top management members early retirement experience: a qualitative analysis}

ENRIQUE CEREZO 1 GABRIELA TOPA ${ }^{2}$

\section{RESUMEN}

Este trabajo analiza, desde un enfoque psicosocial, los discursos sobre su experiencia de prejubilación de una muestra $(\mathrm{N}=7)$ de miembros de Alta dirección. Se utilizó la entrevista en profundidad como técnica de la investigación. Este estudio tenía dos objetivos principales: primero, la identificación de los rasgos de la prejubilación experimentada por los miembros de Alta dirección. Segundo, se buscaba establecer las características distintivas de sus experiencias de control percibido experimentadas durante el proceso. Los resultados muestran la importancia del control personal sobre el proceso de prejubilación y su influencia sobre la calidad de vida posterior al retiro.

\section{ABSTRACT}

From a psychological approach, this paper analyzes the reports of seven top level managers on their early retirement experiences. In-depth interview was used to collect information. The objective of the study is twofold: firstly, to identify the main features of early retirement according to top level managers experience; secondly, an attempt to establish the distinctive features of their experiences of perceived control during the process. Results reveal the relevance of personal control on the early retirement process and its influences on quality of life at postretirement.

\footnotetext{
${ }^{1}$ UNED. Facultad de Psicología.c/ Juan del Rosal, 10.28040 Madrid.psicoeceg@gmail.com

${ }^{2}$ UNED. Departamento de Psicología Social y de las Organizaciones.
} 
La prejubilación en los miembros de alta dirección de las organizaciones: un análisis cualitativo

\section{PALABRAS ClAVE}

Prejubilación, Alta dirección, Investigación cualitativa.

\section{KEY WORDS}

Early retirement, Top management members, Qualitative analysis. 


\section{INTRODUCCIÓN}

La experiencia de la prejubilación se ha presentado durante los últimos años como una posibilidad altamente probable entre el colectivo de personas mayores de 50 años que trabajan por cuenta ajena en alguna de las grandes organizaciones empresariales que operan en España. Según el informe 2/2000 sobre Vida laboral y prejubilaciones del Consejo Económico y Social, con datos de Eurostat, la tasa de actividad media para el conjunto de todas las edades en 1997 era del 55,4\%, mientras que para los mayores de 50 años era tan solo del $28,7 \%$.

Las escasas investigaciones que se han ocupado de las experiencias de la prejubilación desde una perspectiva psicosocial en el ámbito español lo han hecho centrándose en el personal de base de las organizaciones a las que pertenecieron (Alcover y Crego, 2005; Alcover, Crego, Guglielmi y Chiesa, 2009; Crego y Alcover, 2008; Crego, Alcover y Martínez-Iñigo, 2008; Madrid y Garcés, 2000; Martínez, Lozano, Ancizu, Valdés y Candenas, 2003). El colectivo de miembros de la alta dirección de las organizaciones comparte una serie de características, tanto personales como psicosociales, que en conjunto, podrían estar formando un perfil singular en la manera de vivir la experiencia de la prejubilación y que aún no ha sido abordado empíricamente.

El presente trabajo pretende complementar las investigaciones precedentes incorporando el análisis exploratorio de las experiencias de la prejubilación vividas por el colectivo de personas pertenecientes a la alta dirección de las organizaciones. Así, se busca un primer acercamiento a los significados más relevantes de su experiencia de la prejubilación. Además, apoyándose en los trabajos precedentes (Alcover y Crego, 2005; Crego y Alcover, 2008; Crego et al., 2008) se pretende centrar el análisis en el significado de la percepción de control sobre el proceso de prejubilación experimentado por los miembros de la alta dirección de las organizaciones.

\section{La prejubilación: definiciones y justificaciones}

En un prejubilado se dan dos circunstancias: a) el trabajador cesa definitivamente en su actividad profesional remunerada a una edad anterior a la que se fija como edad ordinaria de jubilación para su colectivo; y b) no tiene acceso a una pensión de jubilación, ya sea por jubilación anticipada u ordinaria, tal y como lo define López Cumbre (1991; 1998; 2001).

Las políticas empresariales de prejubilación responden a la creciente exigencia de reducción de costes de estructura organizacional. En los ciclos expansivos de la economía, la frenética carrera por el crecimiento lleva a las organizaciones a buscar este crecimiento a través de fusiones y adquisiciones. El resultado de estos procesos es la duplicidad de muchas funciones y puestos. Una de las formas más habituales, durante los últimos años, de "sanear" las estructuras y reducir el número de recursos humanos han sido los programas de prejubilación. Es decir, facilitar la salida de los empleados mayores, que son los más caros y menos manejables de las organizaciones. En periodos de crisis, como el ciclo en el que se encuentra inmersa la economía mundial en estos 
momentos, la reducción de costes es la prioridad más imperiosa de las organizaciones para intentar sostenerse en el mercado a la espera del cambio de ciclo. Algunas importantes organizaciones multinacionales han anunciado recientemente la reducción de la edad de acceso a sus programas de prejubilación a los 48 años.

Estas justificaciones de los planes de prejubilación son estrictamente economicistas. Están hechas desde la perspectiva organizacional. No contemplan la perspectiva de la persona. Y no se debe ignorar que las personas en los inicios del siglo $\mathrm{XXI}$, en las modernas sociedades desarrolladas, como consecuencia de la mejora de la calidad de vida y el continuo avance de la sanidad, acceden a las edades comprendidas entre los 50 y 64 años en la plenitud de sus condiciones físicas e intelectuales (Freedman, Martin y Schoeni, 2002; Schoeni, Freedman y Martin, 2005).

La prejubilación, por lo tanto, es un fenómeno complejo que implica a la vez aspectos procesales y puntuales. Los primeros están relacionados con la preparación del retiro, en aquellos casos en que tal preparación existe. Los aspectos puntuales están ligados a la decisión de aceptar la jubilación anticipada aquí y ahora. Aunque la prejubilación es frecuentemente vista como un paso abrupto entre un momento de empleo y otro de abandono completo de la actividad laboral, la evidencia sugiere que es una transición más compleja y pausada, al igual que sucede con los procesos de jubilación en general (Pinquart y Schindler, 2007; Wang, 2007). Por otra parte, este proceso tiene un punto clave en la decisión de retiro, que exige considerar la libertad de acción de que disponen las personas a la hora de decidir -de una parte- y -de la otra- los factores push and pull que las llevan a prejubilarse en un momento dado (Szinovacz, 2003). Todos estos elementos condicionan claramente los resultados que se siguen del proceso de prejubilación como avalan diversos estudios empíricos (Davis, 2003; Feldman, 1994; Feldman y Kim, 2003; Kim y Feldman, 2000; Lund y Villadsen, 2005; Quinn y Kozy, 1996; Shultz, Morton, y Weckerle, 1998; Weckerle y Schultz, 1999).

Tomando en cuenta las sugerencias respecto a que la prejubilación implica aspectos procesales y aspectos puntuales (Feldman, 2003; Svinovacz, 2003), la búsqueda de información seguirá una secuencia temporal dividida en: a) la fase anterior a la salida de la organización, b) los momentos inmediatos de la salida, y c) la adaptación a la vida posterior a la salida organizacional. En cada una de estas etapas la búsqueda de información se hará prestando especial atención a los planos macrosocial, meso e individual, según el modelo que sugieren Taylor y Doverspike (2003) para la planificación del retiro.

\section{La alta dirección ante la experiencia de la prejubilación}

El colectivo de personas pertenecientes a la alta dirección de las organizaciones comparte una serie de características que, en conjunto, les distinguen del resto de personas que forman una empresa. Aspectos como la carga e intensidad del trabajo, la duración de la jornada laboral, los niveles de responsabilidad, la habituación a la toma de decisiones, o a la resolución de problemas, la gestión del riesgo, la amplitud de la red social, el nivel de formación 
académica, la capacidad de innovación, el nivel de ingresos o socio-económico, la competitividad, la ambición, el grado de identificación con la organización son, entre otros, algunos de los elementos que caracterizan a las personas que forman parte de los órganos de decisión de las organizaciones (Denis, Lamothe y Langley, 2001; Hambrick, Cho y Chen, 1996; Hambrick y Mason, 1984; Pitcher y Smith, 2001).

En consecuencia, el grupo de los más altos ejecutivos de las empresas tal vez pueda definirse como un "perfil de prejubilados", por su condición derivada del cargo que ostentaban antes de prejubilarse. Además, el interés en el análisis de las experiencias de la alta dirección de las organizaciones se apoya en su capacidad para actuar como modelos de los comportamientos del resto del personal de las organizaciones, en la medida en que los directivos puedan ser valorados por el resto de la organización como "triunfadores" o profesionales con "éxito".

Por otra parte, es necesario considerar que los miembros de la alta dirección pueden mantener un tipo de vinculación con su organización que tenga rasgos distintivos en comparación con el resto del personal. En este sentido, dentro del marco teórico del contrato psicológico, se ha insistido repetidamente en las peculiaridades de tales trabajadores. Así, Hallier y James (1997) han señalado que los directivos son a la vez quienes representan a la organización de cara a los subordinados, pero siguen siendo trabajadores en sus relaciones con la más alta dirección. Así, se ven obligados a mantener un doble papel, porque mientras sirven como agentes del cumplimiento de las promesas de la empresa frente a los trabajadores de base, mantienen sus propias percepciones sobre cómo la organización les trata a ellos mismos en cuanto empleados.

Además, el contenido de sus puestos de trabajo hace altamente probable que se trate de trabajadores del conocimiento, lo cual les añade otra peculiaridad. En su estudio empírico, Flood, Turner, Ramamoorthy y Pearson (2001) encuentran que los trabajadores del conocimiento tienen un amplio abanico de elecciones cuando se trata de escoger empleadores ya que muchas organizaciones compiten por los profesionales de este tipo. Como consecuencia de ello es muy probable que, cuando valoran el trato que reciben de la empresa, este abanico de ofertas esté presente y su umbral de tolerancia ante el incumplimiento de sus expectativas sea más bajo que para otros trabajadores.

Por último, la posesión por parte de estos profesionales de gran cúmulo de conocimientos tácitos hace posible que dicha aportación a la organización sólo esté disponible si el trabajador está altamente comprometido con la empresa. En este sentido, parece que las recompensas y la promoción basadas en el rendimiento tengan un efecto sobre el compromiso y la intención de abandono considerablemente mayor que para los trabajadores de base. En resumen, las condiciones de un "doble" contrato psicológico, sus características como trabajadores del conocimiento y la posesión de un importante cúmulo de conocimientos implícitos pueden conferir a los miembros de alta dirección rasgos peculiares de cara a su manera de preparar y experimentar sus prejubilaciones.

Aunque no siempre sucede es razonable suponer que los directivos pueden 
acceder con más facilidad que otros trabajadores al control de su proceso de prejubilación. El control percibido sobre los acontecimientos de la propia vida tiene un papel destacado en las reacciones de las personas adultas hacia su jubilación (Barnes-Farrell, 2003) y este control del proceso se podría mostrar también como un factor crítico en las reacciones y en los efectos de la experiencia de la prejubilación. En consecuencia, los ejecutivos, como consecuencia de su cargo, podrían disponer de más facilidad para ejercer control sobre el final de sus carreras profesionales por cuenta ajena y, como consecuencia, tendrían una importantísima ventaja frente a otros trabajadores de la empresa en lo que a efectos negativos de la prejubilación se refiere (Szinovac, 2003).

En resumen, debido a la complejidad existente en los procesos de transición dentro de la vida laboral y a las peculiaridades impuestas por la condición de miembros de la alta dirección, en este trabajo se pretende, en primer lugar, explorar los significados más relevantes de la experiencia de la prejubilación vivida por los participantes y, en segundo lugar, centrar el análisis en el significado de la percepción de control sobre el proceso de prejubilación experimentado por estos mismos participantes.

\section{MÉTODO}

\section{Participantes}

Se han realizado siete entrevistas individuales con ex-miembros de alta dirección, con cargos de presidencia o dirección general, pertenecientes a los sectores de servicios profesionales, servicios financieros, energía y telecomunicaciones. El criterio "alta dirección" ha sido establecido exclusivamente desde la perspectiva organizacional, es decir, por el nivel jerárquico en el organigrama de cada compañía. Las organizaciones de las que procedían estos trabajadores son de tamaño muy grande, líderes en sus respectivas actividades y de ámbito multinacional. La organización con menor número de trabajadores emplea a más de 26.000 personas y la mayor supera las 250.000 . La media de edad de los entrevistados es de 56 años, y son todos varones. Este último hecho se debió a la imposibilidad de localizar a miembros de la alta dirección de sexo femenino dispuestos a participar en el estudio. Todos los directivos entrevistados están casados. Cinco de los cónyuges desarrollan una actividad profesional fuera del hogar. Desde el momento de la desvinculación con su organización han transcurrido de media 3,5 años. En todas las organizaciones a las que pertenecían los participantes existían programas de prejubilación. En algunos casos estos programas eran más o menos específicos para los miembros de la alta dirección. Los programas existían con anterioridad a que cualquiera de ellos llegara a la edad de acceso.

\section{Procedimiento de recogida de información}

La entrevista semiestructurada fue preparada previamente según se recomienda (Corbetta, 2007), siguiendo el mapa de categorías construido en base a estudios precedentes (Alcover y Crego, 2005; Crego y Alcover, 2008). Para completar este mapa de categorías se consideraron las sugerencias relativas a los niveles de análisis de Taylor y Doverspike (2003, p. 54) que incluyen un plano macrosocial, uno meso y otro de carácter individual, y 
que en el presente caso se concretaron a través de factores organizacionales, factores grupales y factores personales. Además, este criterio se combinó con la secuencia temporal natural de la experiencia de la prejubilación: anterior al evento, durante el evento y posterior a éste, siguiendo así una perspectiva diacrónica del proceso de prejubilación. Así quedaron establecidas las unidades de contexto (Navarro y Díaz, 1999), fijadas con criterios extra-textuales sobre la base de la información del entrevistador sobre el proceso de prejubilación.

En todas las entrevistas fueron expuestos previamente el objetivo del trabajo, el alcance de la investigación, el perfil de los participantes y la metodología de la investigación. Se pretendió crear un clima discreto, abierto y cómodo, tratando de lograr cierta intimidad, que facilitara la comunicación entre un único entrevistador y un único entrevistado. La duración media de las entrevistas fue aproximadamente de 120 minutos.

\section{Procedimiento de análisis de la información}

Cinco de las entrevistas se grabaron en audio ya que el entrevistado accedió a la solicitud del entrevistador. En las otras dos (D3 y D7) se tomaron notas en forma manual durante el transcurso de la entrevista, debido a que los entrevistados mostraron su reticencia a ser grabados. Posteriormente se transcribió el texto de las notas y de las grabaciones, según correspondiese, para recoger el contenido completo del discurso. El contenido de todas las entrevistas se transcribió en un procesador de texto para PC. El tratamiento subsiguiente de la información se realizó en forma manual sin apoyo de aplicacio-

\section{Tabla 1. Mapa de categorías para el análisis de contenido}

\begin{tabular}{|l|}
\hline Primera fase: situación previa a la salida de la organización \\
\hline a) Causas que desencadenan el proceso de prejubilación. \\
\hline b) Factores que intervienen en la decisión de prejubilación. \\
\hline b1) El significado del trabajo en la vida \\
\hline c) La prejubilación como alternativa esperada o inesperada. \\
\hline Segunda fase: los momentos inmediatos a la salida de la organización \\
\hline a) Dificultades personales en el momento de la salida de la organización. \\
\hline a1) Las relaciones interpersonales durante este período \\
\hline b) El uso del espacio físico \\
\hline c) El desajuste de expectativas \\
\hline d) Las "incompatibilidades" en la prejubilación \\
\hline Tercera fase: consecuencias personales y psicosociales de la experiencia de la prejubilación \\
\hline a) Fuentes de reforzamiento: pérdida de reforzadores \\
\hline b) Las actividades post-laborales. \\
\hline c) Identidad personal, autoconcepto y autoestima \\
\hline d) Relaciones interpersonales. \\
\hline El control sobre el proceso de prejubilación \\
\hline a) Reacciones en procesos de prejubilación controlados por el propio individuo prejubilado \\
\hline b) Reacciones en procesos de prejubilación controlados por la organizaciones \\
\hline
\end{tabular}


nes informáticas específicas para el análisis de contenido textual. El análisis se llevó a cabo según las recomendaciones de Navarro y Díaz (1999). Se establecieron las unidades de registro constituidas por oraciones o párrafos claramente discernibles en base a criterios sintácticos (puntos y aparte), semánticos (cambio de conceptos sobre los que se trata) y pragmáticos (turnos de conversación o cambios de dinámica). En el caso de las dos entrevistas en las que el registro se hizo mediante notas, se aplicó el mismo procedimiento, trabajando sobre el texto del discurso disponible, y empleando primordialmente criterios sintácticos. En el proceso de codificación se pusieron en relación las unidades de registro con las unidades de contexto establecidas en el mapa de categorías en función de su coocurrencia y su intensidad. Esta tarea fue seguida por la integración local, que consistió en la agrupación de todas las unidades de registro relativas a cada unidad de contexto para interpretar el material en forma conjunta (Aguilar, 1999; Valles, 1997).

El procedimiento de codificación se aplicó en forma independiente por los dos investigadores del equipo y se alcanzó un nivel aceptable de acuerdo interjueces $(80 \%)$. Las inconsistencias se resolvieron revisando el mapa de categorías.

\section{RESULTADOS}

La exposición de los resultados del análisis se hará siguiendo el mapa de categorías representado con anterioridad. Un primer análisis de la información de todas las entrevistas nos sugiere la existencia de dos grupos. Parece existir un primer grupo de cinco directivos que, durante los 120 minutos aproximados de la entrevista, no presenta ninguna inconsistencia en sus mensajes, ya sea que su experiencia resulte valorada por ellos mismos como positiva o como negativa. Sin embargo, en el segundo grupo, los otros dos directivos narran sus experiencias, valoran los acontecimientos o describen sus propios sentimientos, pero no siempre parecen hacerlo de forma consistente, sino que su discurso incurre algunas veces en mensajes contradictorios. Esto nos permite hacer una primera agrupación provisional de la información en función de esta diferenciación entre los participantes. Esta diferenciación será aplicada en la primera fase del análisis, referida a la situación previa a la salida, y se retomará parcialmente en el apartado de discusión.

\section{Primera fase: situación previa a la salida de la organización}

Como consecuencia de la existencia de planes de prejubilación formales en todas las empresas a que pertenecían los entrevistados, estos mismos refieren que la experiencia de la prejubilación no fue para ellos algo absolutamente novedoso, desconocido, de lo que nunca hubieran oído hablar o a lo que nunca hubieran dedicado alguna reflexión. Sin embargo, refieren la existencia un hecho desencadenante del proceso.

a) Causas que desencadenan el proceso de prejubilación

Los miembros de la alta dirección describen en su discurso, con mayor o menor claridad, la presencia de un acontecimiento crítico desencadenante. Es el evento causante del cambio que va a justificar el inicio del proceso de prejubilación. Este acontecimiento crítico parece actuar como 
un "marcador psicológico", en el sentido de que los participantes hablan de un "antes" y un "después" de ese momento. Los directivos describen, entre otros, alteraciones en la identidad de la organización, cambios de la cultura de la empresa, en los valores, en la política de gestión de los recursos humanos, cambios en los contenidos, lugares y en los tiempos de trabajo que se suceden a partir de ese momento clave.

En el primer grupo de directivos, aquellos que no presentaron ninguna inconsistencia en sus discursos, no aparece ninguna manifestación de rechazo hacia la organización. No se incluyen valoraciones cualitativas del acontecimiento. Estos directivos no expresan incomprensión. Su discurso es meramente descriptivo. El subyacente economicista en cualquier programa de prejubilación se expone como un elemento que es entendido, compartido y promovido incluso por los propios directivos. El directivo prejubilado ha sido, en ocasiones, el promotor, diseñador, o el responsable de implementar los programas de prejubilación de su organización.

D (Directivo) 3: Yo monté el programa de prejubilaciones para salir a los 55 años y fui el $5^{\circ}$ beneficiado del programa que yo mismo había decidido (...) La base conceptual del modelo es que prejubilando a un alto directivo que gana 100 al que se le sigue pagando por no trabajar en torno a 50 y con los otros 50 se contrata a un profesional mucho más joven. El nuevo profesional llega a tope de ambición y proyección. (...) Es una magnífica decisión.

En el segundo grupo de directivos, en los que sus discursos presentaron inconsistencias, sus narraciones comienzan, al igual que en el primer grupo de altos directivos, con una descripción precisa de su proceso. Sin ninguna carga emocional, sin ninguna valoración cualitativa. Sin embargo en un momento posterior de la entrevista, aparecen mensajes como el siguiente:

D4: ...Amargura la tengo. ¡Joder, he llegado a ser director general! A tener una responsabilidad. A aplicar todo lo que he aprendido. A aplicar toda mi experiencia. Y ahora no cuentan conmigo, ppffff.

b) Factores que intervienen en la decisión de prejubilación

La capacidad de decisión implica la existencia de alternativas y la posibilidad de elección por parte del sujeto entre dichas alternativas. En consecuencia, en este punto sólo se contemplan los factores que intervinieron en la decisión de acceder o no a la prejubilación en los altos directivos que tuvieron dicha capacidad.

En los miembros de la alta dirección de las empresas la decisión de prejubilarse o no parece depender de sus actitudes y valores frente al trabajo. Entre los aspectos que los altos directivos no mencionan en su discurso podemos destacar el momento de desarrollo de la carrera profesional, entendida como la ruptura de una aspiración de subir a un peldaño más alto del organigrama. Tampoco mencionan que influyera en su decisión el compromiso o vinculación con la entidad.

Para los entrevistados el trabajo o, con mayor precisión, el desarrollo de una actividad profesional es un elemento crítico en su planteamiento de vida. El desempeño de un trabajo profesional concreto integrado en una empresa, parece ser sólo un medio específico de satisfacer esa necesi- 
La prejubilación en los miembros de alta dirección de las organizaciones: un análisis cualitativo

dad de estar absolutamente activo. La empresa aparece como un instrumento para lograr desarrollar una actividad.

D1: La necesidad de actividad es total. Para los que hemos trabajado 27 horas al día, la necesidad de trabajo es absoluta. Yo también he tenido y tengo muchas aficiones. Pero no es lo mismo. (...) A los cincuenta y tantos años no puedo entender la vida sin trabajar.

Es el trabajo, entendido genéricamente, no necesariamente el puesto concreto desempeñado en una empresa, lo que parece que actúa como una fuente de identidad, de reforzamiento, de satisfacción vocacional, como fuente de relaciones interpersonales. De hecho, la mayoría de los directivos continúan desarrollando algún tipo de actividad que, además, consideran indispensable que sea recompensada económicamente.

D3: Siempre he tenido claro que había que trabajar. Para mi es fundamental. Y hoy lo tengo todavía más claro, moriré trabajando. (...) Y tener una actividad que además sea remunerada. La parte económica es fundamental.

Los directivos, a pesar de reconocer una situación privilegiada, admitir la cobertura de sus necesidades económicas y disponer de un patrimonio que les otorga una buena protección ante los posibles riesgos imprevistos del futuro, atribuyen una gran importancia a los aspectos económicos. Además de considerar la situación económica previa con la que se llega al momento de tomar la decisión sobre la permanencia o la salida de la organización, los directivos también incorporan la necesidad de continuar generando ingresos tras su salida de la vida laboral por cuenta ajena.
El hecho de que el trabajo pueda ser considerado una forma de vida no parece ser un factor que desaliente la salida de la organización.

D5: La actividad tiene que tener una contraprestación por ambas partes. En el mundo de la empresa, lo que es gratis no tiene valor. Lo que haces creo que tiene que tener un justiprecio. (...) Para mi eso es esencial.

Para un único directivo entrevistado el trabajo es considerado exclusivamente como un medio de vida:

D4: Yo he trabajado para vivir y no he vivido para trabajar. Todo mi esfuerzo ha sido para poder vivir y poder vivir de una forma desahogada. Poder tener una casa, pagar la hipoteca, poder pagar un coche, tener hijos, buenas vacaciones...

Y este directivo es el único de los siete que no desarrolla ninguna actividad profesional.

D4: Yo he trabajado cuarenta años. Nunca he tenido tiempo para aburrirme. (...) Mi ilusión durante los últimos años era tener tiempo para aburrirme.

La valoración personal sobre la tarea es también un factor clave en los directivos a la hora de decidir sobre la permanencia o deseo de salida de la organización:

D2: Llega un momento de la vida en la que te das cuenta que la limitación mayor que tiene la vida es el tiempo. Y es muy importante dedicar el tiempo que te quede a las personas y a las cosas que realmente creas que merece la pena dedicar el tiempo. Entonces ¿Qué adelantaba yo siguiendo en (nombre de la empresa) durante 10 años más haciendo lo mismo? 
D3: Después de más de 30 años de carrera profesional yo estaba harto de mi trabajo. Yo necesitaba un cambio. Dar un giro profesional. Un cambio de vida radical.

D5: Vi la oportunidad, el panorama y la oferta que me habían hecho, y bueno, yo puedo seguir de (cargo de alta dirección) y ser (mayor cargo de alta dirección) en dos o tres años más. Pero yo tenía la sensación que cuando me levantaba todas las mañanas, todos los días, como que la película ya la había visto. Mi sensación es que había llegado el momento de rentabilizar los muchos años de trabajo.

Los directivos entrevistados para este trabajo manifestaron experiencias valoradas como monótonas o poco motivadoras, ya que el aburrimiento y la caída de la motivación parece que actúan como desencadenantes de una decisión de salida de la organización en su caso. Los directivos mencionaron una reivindicación de cierta recuperación de su libertad, de soberanía sobre su propio tiempo y su propio destino.

c) La prejubilación como alternativa esperada o inesperada

En el caso de que se produzca una percepción de desajuste entre la fecha esperada de salida y la impuesta por la organización, los altos directivos reaccionan rechazando la anticipación abiertamente.

D1: ...entonces, como me preguntabas, yo tenía asumido que a los 55 me llegaría el momento de salir. Pero me tocó a los 52 . Me llaman un día y me dicen: oye (su propio nombre) que te toca.

¿Sorprendido? Me pilló sin plan B. Fue una gran sorpresa para mí.
Los discursos ponen de manifiesto que, en tales casos, experimentan un proceso de prejubilación no previsto que complica las reacciones de las personas implicadas.

D1: (...) la verdad es que cuando me llamaron a mí no me lo esperaba. A mí no me tocaba. No tenía plan B. Me pilló sin recursos.

Esperaba que fuese más tarde. Tres años más tarde.

\section{Segunda fase: los momentos inmediatos a la salida de la organización}

La segunda fase del proceso de prejubilación corresponde a los momentos en los que se materializa de forma efectiva la salida de la organización. Sin duda, a pesar de que este periodo es el más breve de las tres fases temporales identificadas en la experiencia de la prejubilación, en muchas ocasiones parece ser la fase que implica una mayor carga emocional y mayores tasas de estrés. Esto quedó patente en los discursos de los entrevistados. Mientras que sus experiencias durante la primera fase de las entrevistas fueron contadas de forma descriptiva, en esta segunda parte, los directivos compartieron sus experiencias más personales, sus reflexiones y emociones más íntimas, con lo que se potenció la importancia del lenguaje no verbal.

a) Dificultades personales en el momento de la salida de la organización

Los directivos pusieron de manifiesto algunos acontecimientos de elevada tensión emocional en las semanas o meses previos a abandonar la organización. Se les exigía soportar niveles de estrés muy 
altos hasta el último día. Se trataba de un periodo de tiempo extraordinario, fuera de la rutina habitual pero con una alta carga emocional. A los directivos, en las semanas o meses previos a abandonar definitivamente la organización, se les presentan numerosas situaciones confusas.

D3: Es curiosa la reacción de la gente. Es como si tuvieras una bota apoyada en el suelo sobre una balsa de aceite. Levantas la bota y el aceite empieza a cubrir el espacio que tu has dejado al levantar la bota y tu huella es rápidamente cubierta por el aceite.

Las relaciones interpersonales dentro de la organización muestran nuevas dimensiones desconocidas hasta ese momento. Aparecen intereses y ambiciones de compañeros que habían estado ocultas. En esos últimos momentos se sufre una situación de indefinición y de indefensión, cuando menos incómoda.

D3: El resto de la organización ocupa el lugar que tú tenías y tu huella desaparece más rápido de lo que tú te hubieras imaginado. Incluso antes de que abandonara la compañía, mis propios compañeros se iban haciendo hueco y ocupando mi sitio, cubriendo la huella.

\section{b) El uso del espacio físico}

Un aspecto importante en el análisis de las reacciones inmediatas de la experiencia de la prejubilación es el lugar en el se está físicamente.

D3: ...cuando la universidad no me sirvió y falló, "trabajé" durante meses en (nombre de una cafetería) de la calle (nombre de la calle). Tenía una mesa fija donde pasaba las mañanas diseñando lo que quería hacer después de fracasar el proyecto de la universidad. Me reunía con gente en la misma cafetería. Pero siempre desde fuera de casa.

En sus discursos, los directivos dan bastante importancia al hecho de que se deja de ir a la oficina, se abandona el despacho y se carece de un lugar diferente al hogar en el que pasar el día.

D2: Yo tengo una oficina. Tengo una oficina junto a mi casa. Entre otras cosas porque lo necesito. Tengo una oficina propia junto a mi casa en un edificio nuevo que me han entregado hace un año. Hasta ese momento he estado instalado en oficinas de amigos, muy caritativos, que me han dejado un espacio para poder trabajar, tener mis reuniones o recibir a mis clientes.

c) El desajuste de expectativas

El equilibrio personal y el orden vital que el prejubilado dice haber conseguido después de la salida pueden resultar estables. En ocasiones, por el contrario, puede ser tan sólo un escenario establecido inmediatamente después de la salida, pero que se desmorona por cualquier circunstancia. Se produce así un desajuste entre las expectativas que el prejubilado había construido sobre el momento posterior a la salida y la realidad con que se encuentra. Ante ello se ponen de manifiesto diferentes estrategias de afrontamiento. En algunos casos, los esquemas cognitivos que sustentaban la situación post-laboral se mostraron débiles y acabaron por venirse abajo ante la aparición de eventos imprevistos.

D1: Lo que además me ha sucedido a mí, es que me ha sido muy difícil gestionar la prejubilación con las "incompatibilidades" 
(...). Resulta que lo que has hecho durante toda tu vida, lo que mejor sabes hacer, lo que puede que sea lo único que realmente sabes hacer, es justo lo que no te dejan seguir haciendo.

Por el contrario, también puede suceder que a pesar de la pérdida de los elementos en los que se apoya la estrategia post-laboral, los esquemas cognitivos se muestren fuertes y se venzan las dificultades que puedan aparecer.

D3: Cuando la universidad no me sirvió y falló, "trabajé" durante meses en (nombre de una cafetería) de la calle (nombre de la calle). Tenía una mesa fija donde pasaba las mañanas diseñando lo que quería hacer después de fracasar el proyecto de la universidad.

... La verdad es que yo soy bastante "enreda". Soy bastante inquieto. Me gusta ver donde están las oportunidades de entrar barato y salir más alto. (...) Desde entonces, tengo oficina abierta en Madrid, con varias personas, desde donde llevamos el proyecto.

d) Las incompatibilidades durante la prejubilación

En la prejubilación de la alta dirección, además, se establecen con frecuencia cláusulas de "incompatibilidades". Éstas, a cambio de una compensación económica, impiden al ex-directivo desarrollar determinadas actividades en los sectores afines a la organización o le imponen la obligación de solicitar autorización a la exempresa para el desarrollo de cualquier actividad.

A los efectos de la prejubilación no esperada, la que llega antes de tiempo, por sorpresa, se le une con frecuencia, la inmovilización que representa la cláusula de "incompatibilidades". Esto parece sumarse al desajuste de expectativas de que se habló anteriormente y algunos refieren una reacción de desorientación.

D1: Yo no sé si uno puede prepararse para la prejubilación. Creo que no. Lo que creo es que hay que anticiparse. Hay que tener previsto un plan B. (...)

Creo que la prejubilación sin una condición de "incompatibilidades" es una situación diferente. Eres más mayor, si, pero aquí estoy yo, con ganas, dispuesto a afrontar el reto. Lo que sucede es que por una evaluación económica aceptas las "incompatibilidades" y la situación posterior es muy difícil, muy difícil (...) Además como has firmado "incompatibilidades"... Tú encuentras algo que te interesa, pero las "incompatibilidades" te lo impiden hacer. De repente no tienes historia profesional.

\section{Tercera fase: consecuencias personales y psicosociales de la experiencia de la prejubilación}

En la muestra de directivos utilizada en este informe se pueden distinguir dos grupos en función de las consecuencias percibidas de la prejubilación. Por una parte podríamos hablar de aquellos individuos que, tras la salida de la organización, tienen que implementar las actividades y comportamientos planificados previamente. Por otra parte, aquellos individuos que han pasado por una fase de "duelo" y una posterior reorganización de su vida post-laboral.

Todos los directivos entrevistados, excepto uno, manifestaron con rotundi- 
La prejubilación en los miembros de alta dirección de las organizaciones: un análisis cualitativo

dad la necesidad de continuar desarrollando actividades retribuidas económicamente.

\section{a) Fuentes de reforzamiento}

Los altos ejecutivos de grandes empresas que hemos entrevistado consideran que accedieron a sus puestos de máxima responsabilidad combinando determinadas habilidades y capacidades personales con enormes dosis de esfuerzo. Alcanzar los niveles más altos de las organizaciones era para ellos una fuente de satisfacción por el logro de metas tan ambiciosas además del reforzamiento social que acompañaba a esos cargos. Igualmente, el nivel de las relaciones sociales que se establecen en los puestos de la alta dirección era otra importante fuente de reforzamiento.

En consecuencia, algunos de estos directivos afirman que la prejubilación les supuso la pérdida del reforzamiento vinculado a su elevada posición en el organigrama de la organización.

D1: Bueno, tú sabes que uno disfruta de las cosas en la medida en que las dominas, (...) Claro, cuando (...) de repente tienes que empezar una actividad totalmente nueva a los cincuenta y tantos años, en la que no eres nadie, ni sabes nada, pues es muy duro. Créeme que es algo muy duro.

Claro, también tiene otro lado: te rejuvenece. Te hacer sentir activo y vigente.

D4: Yo entré de administrativo y he terminado de director general. Es una gran satisfacción. Pero tengo un gran amargor. (...) He hecho las cosas bien y la empresa, la gente (...) decide que no... que ya has aportado lo que tenías que aportar. Que no le importa si tenías que aportar algo más.
Sin embargo, también se pueden encontrar altos directivos que manifiestan una identidad personal más independiente de las fuentes de reforzamiento asociadas a la organización o al cargo que ostentaban.

D2: Yo soy una persona que no valoro mucho el despacho, las secretarias, los coches de empresa, el boato, las decisiones de poder. No me producen, ninguna satisfacción especial, ni tampoco ninguna dependencia en absoluto. A mí lo que me ha dado satisfacción personal han sido los proyectos en los que he participado, que todos han sido muy importantes y enormemente atractivos. Yo no he sido más feliz por ser director general de esto o de aquello.

D6: Y, en mi caso, la autoestima no baja. A mi no tener poder no me ha resultado ningún trauma. En absoluto. (...) El día que consiga abrir mi clínica personal, y ponerme a trabajar eso va a ser el autorrefuerzo mundial. Y que tenga algún cliente. (...) El primer tío que entre con depresión y que salga contento, eso puede ser la de Dios Entonces ya si que puedes decir, es que soy Dios, me lo creía por los libros, pero es que yo soy capaz de ayudar.

\section{b) Las actividades post-laborales}

Los miembros de la alta dirección habituados a unos programas de actividad muy intensos, con agendas apretadísimas y horarios interminables pueden sufrir de manera más intensa los efectos de la desaparición de las obligaciones laborales cotidianas.

D4: El cambio es brutal. El cambio es brutal. Y si no estás concienciado, más todavía. Yo me busqué una distracción. Yo me metí en el jardín y lo cambié entero. (...) Los primeros días el móvil sigues mirándo- 
lo. Tienes unas sensaciones raras. El mono del ordenador. (...) Esas sensaciones los primeros días, sí las tienes, pero a mí, a mí, me duraron poco. (...) Ya no echaba de menos el ordenador, ni...

Los directivos, a través de estrategias más o menos elaboradas, pero con compromiso y disciplina, afrontan la desaparición de sus apretadas jornadas laborales y la pérdida de numerosas rutinas asociadas al desempeño de determinados puestos en una organización

D4: ...De hecho, me tiré prácticamente seis meses sin tocar el ordenador de mi casa. Me lo planteé como una terapia, como una droga. Yo creo que hay cosas que te enganchan psicológicamente... ....y cuando digo lo dejo, lo dejo. Como una droga. Se acabó. (...) Otra gente se come el coco. Tomas una decisión y ya no puedes cuestionarte más cual habría sido tu vida.

Esto lo he hecho para protegerme. Tenía miedo de que realmente sufriera mono. Después de estar catorce horas diarias trabajando, estar todo el día al ordenador y al móvil y eso me pasara factura después.

c) Identidad personal, autoconcepto y autoestima

Algunos entrevistados contemplan al trabajo como la fuente de la identidad de la persona y como un elemento crítico en el auto-desarrollo personal. El alto directivo también señala la existencia de pérdidas personales:

D1: Sobre el papel, públicamente, mi situación puede ser bastante atractiva. (...) Entonces, me podrías preguntar: ¿qué más quieres? Pero bueno, dicho esto, y en esta vocación de sinceridad que me has pedido, yo no estoy nada satisfecho. Porque, primero, he perdido mi agenda. De ser una persona que estaba en la pomada, en todas las movidas, de repente te quedas sin nada. Psicológicamente es muy impactante (...) De repente no tienes historia profesional. Es como si hubieras tirado tu currículum vitae.

Las reacciones de la anterior transcripción muestran la importancia para la identidad y estabilidad personal de los elementos profesionales asociados a un cargo de alta dirección. La decisión sobre la prejubilación, cuando el trabajo actúa como soporte de la identidad personal, es el rechazo ante la amenaza de pérdida de las fuentes de dicha identidad que acompañaría a este acontecimiento.

D1: De repente (...) Te quedas sin poder utilizar tus habilidades, tu capacidad mental, lo que has hecho toda tu vida, lo que mejor haces. ¡Que por eso has llegado a lo más alto!

Aquí se pone de manifiesto en el discurso de los entrevistados la diferencia entre dos tipos de procesos de prejubilación, aquellos que han acabado deteriorando la autoestima de los entrevistados y los que no lo han hecho.

D2: Yo no he vivido o sentido nada especial en relación a mi autoestima. La verdad es que yo me he sentido mejor conmigo mismo cuando he sido capaz de abordar determinadas cosas que anteriormente no hacía. A mí me gusta mucho más aprender que hacer. Y desde que estoy prejubilado he aprendido muchísimas cosas y eso me reporta una enorme satisfacción.

Sin embargo, en otros casos de experiencias de prejubilación se produce un deterioro claro de la autoestima. 
La prejubilación en los miembros de alta dirección de las organizaciones: un análisis cualitativo

D1: La pregunta es ¿En qué me he equivocado? ¿Cómo no lo he previsto? ¿En qué me he equivocado para no haber previsto todo esto? ¿Por qué no he tenido un plan B preparado?

Por qué no he reaccionado y les he dicho: echadme joder. Echadme a la calle, pero dejadme libre. Echadme a la puta calle pero dejadme moverme...

...hay veces que ves a tíos que son mayores que yo, y que no son mejores que yo, y siguen trabajando en una compañía, y yo no. ¿Y qué es lo que he hecho yo mal?

D4: ¿Pero me voy a pegar un tiro por eso? Peor para ellos que se han perdido una oportunidad de tener a alguien que sea capaz de... capaz... de ayudarles. Nada más. Capaz de ayudarles. ¿Qué prescinden de mí? Es su problema.

\section{d) Relaciones interpersonales}

En el ámbito de las relaciones interpersonales se pueden contemplar dos áreas de interés. Por una parte las relaciones familiares, especialmente la pareja y por otra parte las relaciones extrafamiliares.

El punto más destacable en el área de las relaciones interpersonales vinculadas a la experiencia de la prejubilación expresado explícitamente por los nuestros participantes, es la absoluta independencia y autonomía que pusieron todos de manifiesto en el momento de la toma de la decisión definitiva. La prejubilación es valorada como una experiencia individual. Como algo perteneciente al ámbito privado del individuo. Es una responsabilidad individual. No es un problema de la pareja, tampoco es un problema de los hijos.

D1: ...pero eso no es un problema, Eso no ha sido un problema para mí. El problema no son ellos (refiriéndose a su familia). El problema no es de ellos. El problema es mío. El problema es personal.

Tú te sientes joven y bien, y te quedas en casa. Tus hijos salen a sus ocupaciones por la mañana, y tú te quedas en casa. No es agradable. Es como que eso no te toca. Pero es más tu sensación. Ellos (los hijos) lo asumen bien. Es sólo tu problema, para ellos tu prejubilación no es un problema.

D2: En mi caso, el acierto o el error de mi decisión, es sólo mío. Aunque creo absolutamente que acerté. Pero en cualquier caso, el acierto o el error hubiera sido mío. Por supuesto no es de la empresa. Toda la iniciativa de mi prejubilación fue mía. La empresa se portó muy correctamente conmigo.

D3: La prejubilación es una experiencia personal. Hay que gestionarla solo.

D5: Por supuesto mi escenario es el que es. Cada uno tiene que resolver la ecuación de su vida con las variables que tiene. No todas las ecuaciones son las mismas. Algunos escenarios de algunas personas son muy complejos.

D7: Sin duda piensas en tu mujer o en tus hijos. Pero la decisión lo tomas tu solo. Como un cambio de trabajo, tus cargas siempre las tienes en tu análisis pero la decisión es tuya. La tomas tú sólo.

El papel de los hijos prácticamente no se pone de manifiesto. Sin embargo, la relación con la pareja sí que fue abordada 
en las intervenciones de los directivos. En los discursos se insiste en la exigencia de neutralidad de la pareja en el proceso de la prejubilación. Una neutralidad de enorme importancia. Los ejecutivos reconocieron el trascendente papel de sus parejas; pero además, a la pareja se le reconoce la capacidad de hacer daño y de interferir en la estabilidad emocional. La postura de la pareja puede agudizar el estado de bloqueo y de desequilibrio en los momentos críticos del proceso.

D3: En otro momento, en los inicios de la prejubilación, recuerdo que ella me hacía muchas preguntas, cuestionaba mucho lo que había hecho, lo que estaba haciendo. Yo soy bastante fuerte, pero en un momento tuve que parar el tema. Una cosa es ser fuerte, tener las cosas muy claras y otra que tu mujer te toque los coj... Para muchas de las cosas que he ido viviendo desde que salí de mi empresa estaba preparado, pero no para que me toquen los coj... en mi propia casa.

Los entrevistados plantean también el tema de las dobles carreras, las mujeres continúan trabajando en la mayoría de los casos. Pese a este hecho, no lo han mencionado como un problema.

D2: El tener una oficina propia fuera de casa nos permite tener una vida muy ordenada. Mi mujer y yo tenemos nuestras propias ocupaciones y nuestros propios espacios. Esto contrariamente a lo que pueda parecer, lo que provoca es que tratamos de coincidir en lo que podemos. Si puedo la llevo yo al gimnasio, tratamos de comer juntos, etc.

Por ultimo, en el ámbito de las relaciones extra-familiares, los directivos pusieron de manifiesto una valoración de las relaciones sociales ligadas al entorno pro- fesional carentes de profundidad o de intimidad. Los "amigos", o mejor dicho compañeros, establecidos en el ámbito profesional se diluyen inmediatamente y para ninguno de los directivos supuso ninguna sorpresa. Fue una valoración compartida por todos los entrevistados.

Por otra parte, en el caso de alguna experiencia de prejubilación más negativa (D1, por ejemplo), la percepción de soledad se puso explícitamente de manifiesto. Muchos de los directivos que viven su experiencia de forma inesperada y mencionan que hubieran querido contar con un entorno afectivo próximo que estuviera viviendo su misma experiencia. Hubieran deseado compañía, complicidad en su proceso.

\section{El control sobre el proceso de prejubilación}

El último apartado del análisis se centra en una exploración más detallada de las percepciones de control de los entrevistados sobre su proceso de prejubilación. En el análisis de esta categoría se ha seguido una perspectiva sincrónica, ya que no se han contemplado por separado los diferentes momentos temporales sino que se ha analizado la percepción de control en forma general.

En cuanto al control sobre los aspectos implicados en el proceso de la prejubilación, se pueden distinguir dos grupos de participantes. Un primer grupo de directivos de los que se puede concluir, a través de sus testimonios, que mantuvieron la iniciativa en sus procesos, tanto en cuanto a la elección del momento como de las condiciones en que se produjo. En estos casos, ellos se perciben a sí mismos como 
La prejubilación en los miembros de alta dirección de las organizaciones: un análisis cualitativo

dominadores de la situación. Controlaron el tiempo y las condiciones.

D3: En mi caso, yo he controlado totalmente la situación. El programa de prejubilaciones lo diseñé yo y lo implanté yo. Sabía perfectamente el cómo, cuándo y de que manera.

En el caso de mi prejubilación, sabía que debía dominar la situación, tomar yo las decisiones...

Para mi es fundamental la diferencia entre voluntaria o forzosa. (...) Para mi fue, sin ninguna duda, la oportunidad de dar un cambio radical a mi vida. Era lo que yo deseaba y lo hice cuando yo quise.

D6: ...la oferta de (ciudad europea) significaba que si aceptaba esa alternativa, volvíamos a impulsar mi carrera profesional, y para mi no iba a haber prejubilación. Y es entonces cuando rechazo (ciudad europea). Por mi, yo no quería pero, para mi familia, tampoco. Yo no me quería ir a (ciudad europea)... (...) El caso es que sigo activo pero no tanto como antes. Ya no podía ascender más. (...) Entonces, encantado con mis estudios de la carrera de psicología, y con las ideas muy claras, solicito prejubilarme dos años antes. Mi jefe me pide que no lo haga. (...) El caso es que aguanté hasta diciembre de 2004, y consigo prejubilarme con 10 meses de adelanto sobre lo que me correspondería y me prejubilo encantado.

Esta última afirmación sugiere que el control sobre el proceso de la prejubilación parece, pues, consistir en un elemento clave, especialmente si se atiende a los efectos que la experiencia de la prejubilación produce sobre las personas.

En un segundo grupo de directivos no se puede interpretar con igual claridad quién mantiene el control del proceso: si el directivo o la organización. En sus relatos se mezclan elementos, resultando que el control parece que está en manos de la empresa por momentos:

D5: Claro que me la esperaba (la prejubilación). Por muy alto directivo que seas, al final formas parte de una hoja Excel, con unos números, con unos datos, donde se cuantifica la situación. (...) Al final en los números de la empresa tú tienes que restar para que los números se cumplan. (...) Eres un número.

Pero en otros momentos, el mismo entrevistado parece tener en sus manos el control de la situación:

D5: ¿Podría haber seguido? Si claro. Pero a lo mejor pasan unos años y llega otro plan en el que no te dejan elegir y te tienes que ir, si o si, y entonces las condiciones ya no son como las de ahora. Con lo cual no podía dejar de aprovechar las ventajas o los derechos que si se me reconocían en el plan que me tocaba. Ahí reaccione muy objetivamente. Muy racionalmente....

... El control es total de la compañía. Sólo en el momento de la decisión el control lo tienes tú. Durante muy poquito tiempo. Pero si no tomas la decisión, y la decisión que espera la compañía, la empresa la toma por ti. Entonces yo me dije, pues si la decisión la tengo que tomar yo, la tomo yo. Tuve el control poco tiempo pero el suficiente para hacerme una composición de lugar.

En resumen, entre la alta dirección que se ha entrevistado en este trabajo se pueden identificar dos tipos de situaciones en relación con el control mantenido sobre el 
proceso de prejubilación. La primera correspondería a los directivos que han dominado o controlado la mayoría de los aspectos asociados a la decisión sobre su prejubilación. A juicio de los entrevistados, es la previsión, la completa y correcta planificación y el control del proceso lo que les permitirá vivir la experiencia de la prejubilación como una oportunidad en lugar de una amenaza. La segunda situación corresponde a los directivos a los que la prejubilación les sobrevino de manera totalmente imprevista, sin preparación alguna, como una imposición. Las reacciones de estos directivos están descriptas como plagadas de muchas dificultades para dominar el proceso de la prejubilación. Expondremos a continuación ambos conjuntos de respuestas por separado:

a) Reacciones en procesos de prejubilación controlados por el propio individuo prejubilado

b) Reacciones en procesos de prejubilación controlados por las organizaciones.

a) Reacciones en procesos de prejubilación controlados por el propio individuo prejubilado

Los directivos que exponen en sus discursos que controlaron las decisiones y los procesos asociados a su propia prejubilación, manifestaron reacciones racional y emocionalmente equilibradas. El tránsito del trabajo por cuenta ajena al desarrollo de otras actividades fuera de una gran empresa parecía responder a un deseo profundo, voluntario, y largamente meditado. Planificaron cuidadosamente su proceso de prejubilación. Se preocuparon de prever las actividades inmediatas tras la salida de la organización. No se detectó ninguna improvisación en sus decisiones.
D3: Yo preparé la salida de la sociedad concienzudamente. No he improvisado nada. Lo primero es que sabía que no podía quedarme en casa. Cuando decidí que dejaba la empresa, incluso antes de abandonarla, empecé a trabajar en la universidad privada (nombre de la universidad). (...) y planeé con mucho detalle como lo iba a hacer. En la actualidad tengo varios consejos de administración, como consejero independiente y colaboro con algunas empresas en proyectos concretos...

D7: Lo que pasa después de prejubilarte es consecuencia de un proceso mental de programación y de profundo conocimiento personal y de las condiciones de los mercados profesionales para anticipar tus verdaderas posibilidades fuera de (nombre de la organización de salida). Es decir, ahora he constituido una empresa a nombre de mi mujer a través de la cual sigo trabajando para (nombre de la organización de salida). Tengo varios cargos en diferentes consejos de administración, que he formalizado tras mi salida de (nombre de la organización de salida), pero que ya tenía negociados y apalabrados antes de prejubilarme. Estoy dando clases en una escuela de negocios. Vamos, que no he improvisado lo que me está sucediendo. Que hay una labor previa que hacer antes de tomar la decisión de prejubilarte.

D6: ...entonces empecé a pensar que si me van a empujar para que me vaya, yo la verdad es que no sabía si quería irme o no quería irme, pero lo que si podía hacer era empezar a interesarme por algo, y más que interesarme, a emocionarme con algo... ...pero sobre todo, ya había interiorizado la oportunidad que tenía de cambiar mi vida. Ya no quiero ser más ingeniero, quiero hacer otra cosa... 
La prejubilación en los miembros de alta dirección de las organizaciones: un análisis cualitativo

b) Reacciones en procesos de prejubilación controlados por la organización

En el caso en que la prejubilación aparece de forma imprevista, sin tiempo de planificar o programar la situación se puede producir una importante desorientación, falta de iniciativas capaces de organizar la vida fuera del trabajo en una organización.

Cuando la prejubilación se presenta de forma inesperada, sin opciones para tomar ninguna decisión, la vivencia de dicha experiencia parece tener consecuencias devastadoras.

D1: ...Fue una gran sorpresa para mí. Asumiendo que eran las reglas del juego, asumiendo también el concepto, admitiendo que yo estaba de acuerdo con la existencia de un programa de prejubilaciones, la verdad es que cuando me llamaron a mí no me lo esperaba. (...) Yo no sé si para la prejubilación uno puede prepararse. Creo que no. Lo que creo es que hay que anticiparse. Hay que tener previsto un plan B. (...)

\section{(Entrevistador): ¿La clave podría ser la falta de control del proceso?}

\footnotetext{
Absolutamente. Ese es el tema. (...) Pero las consecuencias posteriores no las valoras correctamente. No te las planteas. Es tu empresa de toda la vida, tienes una buena relación..., de alguna manera siempre había aceptado todo lo que la empresa me había ido proponiendo y siempre me había ido bien. No prevés la situación posterior.
}

\section{DISCUSIÓN}

A través del análisis de las entrevistas mantenidas con siete altos directivos de organizaciones empresariales se ha pretendido explorar los significados asociados a la prejubilación en este colectivo específico y así complementar los trabajos realizados con miembros del personal de base de las organizaciones, recogido en trabajos precedentes (Alcover y Crego, 2005; Crego y Alcover, 2008; Crego et al., 2008). En este primer acercamiento exploratorio a las vivencias de los miembros de la alta dirección nos proponíamos acceder a sus percepciones durante todo el proceso, haciendo especial hincapié en el control percibido por los implicados sobre los acontecimientos y en las posibles consecuencias de esta percepción de control o de su ausencia.

En nuestro estudio se ha verificado, pese a las limitaciones de la muestra que se comentarán más adelante, la influencia positiva que la preparación del retiro tienen para el bienestar de quienes se jubilan anticipadamente, al igual que el hecho de que los programas de preparación podrían incrementar el número de personas que acepten esta alternativa (Feldman, 2003). En este sentido, los prejubilados que preveían la situación y pudieron organizarse para hacerle frente se encuentran en mejores condiciones vitales y, en algunos casos, se consideran a sí mismos como los máximos beneficiados de la situación, puesto que les ha permitido reorientar sus vidas hacia actividades más atractivas.

No obstante esto, también se aporta evidencia coherente con los modelos de continuidad (Atchley, 1989), ya que se manifiesta que aquellos directivos con posibilidades de continuar desarrollando actividades profesionales retribuidas económicamente son los que se muestran más satisfechos en líneas generales. A pesar de acceder a la prejubilación con 
sus situaciones económicas resueltas, los seis coincidieron, no sólo en su deseo de continuar haciendo cosas, sino también en la necesidad de que éstas estuvieran económicamente remuneradas. Tal vez para los directivos los conceptos de actividad asociada a rendimiento o de logro y utilidad, típicos de la sociedad occidental desarrollada, estén más fuertemente arraigados en sus modelos de vida. Esta evidencia es coherente con estudios recientes en el marco europeo que insisten en los efectos positivos que los empleos puente tienen sobre el bienestar de las personas mayores (Topa, Moriano, Depolo y Morales, 2009).

Con frecuencia se hace referencia a la prejubilación de los directivos como una situación idílica respecto a la cual no hay quejas legítimas. Sin embargo, algunos de nuestros participantes no lo experimentan así. Esta evidencia podría estar apoyando la sugerencia de otros autores (Wang, 2007; Hobfoll, 2002) respecto a la conveniencia de considerar la transición desde una perspectiva de disponibilidad de recursos, en el sentido de posibilidad de satisfacer las necesidades vitales. Así, habría que tomar en cuenta las capacidades físicas (McArdle, Vasilaki, y Jackson, 2002), los recursos cognitivos (Park, 2000), los motivacionales y los financieros (Taylor y Doverspike, 2003) al igual que los sociales (Kim y Feldman, 2000). Con este abanico amplio de indicadores se puede valorar más adecuadamente la prejubilación, al igual que la jubilación, como un momento de cambio en los recursos disponibles. Por lo tanto, la prejubilación puede tener un impacto negativo en el bienestar, no tanto por la cantidad total de recursos de que se dispone sino más bien por la discrepancia entre el momento anterior y el posterior a la salida.
Entre nuestros entrevistados también se encuentra evidencia coherente con el hecho de que las disrupciones bruscas de los ritmos vitales, de los estilos de vida arraigados en el grupo de directivos, tales como contar con el espacio de una oficina, con personal de asistencia administrativa exclusivamente a su servicio, son fuentes de estrés y de consiguiente malestar (Szinovacz, 2003). En concreto, entre nuestros entrevistados hay algunos que han pasado por una fase de "duelo" y una posterior reorganización de su vida post-laboral. Esta situación parece que está asociada al control del proceso de transición de la organización a las actividades post-laborales privadas o al acceso a la experiencia de prejubilación de forma no esperada, no planificada, como se comentará más adelante. En este sentido se recoge información que avala las funciones psicosociales del trabajo, señaladas hace ya tiempo (Peiró y Prieto, 1996), entre las que destaca el papel del trabajo como estructurador del tiempo y de la actividad cotidiana. En parte, nuestra muestra pone de manifiesto que, incluso en aquellos casos que la prejubilación sobrevino de forma inesperada, la recuperación del bienestar personal depende aún de la recuperación del control, del dominio sobre la propia situación vital. Así lo manifiestan quienes narran que no tenían plan $B$ o quien dice que tuvo que trasladar su despacho a una famosa cafetería de Madrid.

En resumen, esta evidencia parece coherente con el hecho de que la prejubilación pueda tener diferentes patrones de salida, al igual que lo tiene la jubilación, como se ha mostrado recientemente en un estudio longitudinal con una muestra procedente de la encuesta Health and Retirement Survey (Wang, 2007). En el referido trabajo se encontraron tres patrones, de los 
cuales uno era de mantenimiento, otro de recuperación y el tercero tenía forma de U. El primero de ellos era el más probable en el caso de los jubilados que conservaron empleos puente, planificaron activamente su salida y tenían matrimonios felices cuyas esposas no trabajaban. El último era el más probable para quienes se retiraron prematuramente, entre otras características. Esto parece coincidente con nuestros participantes, ya que quienes han logrado conservar sus actividades profesionales más allá de la prejubilación y fueron eficaces en la preparación de su retiro no mostraron grandes insatisfacciones con la situación presente. Por el contrario, aquellos que fueron sorprendidos por la prejubilación, que la consideraron prematura o no se hallaban preparados, experimentaron inicialmente un declive de su bienestar y tuvieron luego que hacer un esfuerzo mayor para recuperarse.

En relación con este mismo punto, la literatura precedente señala que las transiciones tendrán un efecto más negativo sobre el bienestar cuando ocurran a destiempo (Szinovacz, 1989; 2003) y este hecho se constata entre nuestros entrevistados, en general, puesto que la prejubilación es un retiro anticipando en sí mismo $\mathrm{y}$, en especial, en quienes no esperaban prejubilarse tan pronto. Al mismo tiempo, los directivos aportan evidencia que sostiene la existencia de discriminaciones en función de su edad en las organizaciones, lo cual es también coherente con la literatura precedente (Feldman, 2003), especialmente en lo relativo al concepto de edad normativa para el retiro de la vida activa (Cleveland y Shore, 1992).

La cuestión más relevante que se ha podido recoger en esta primera aproximación exploratoria a los discursos de los prejubilados de alta dirección es la importancia de la percepción del control que la persona ha mantenido durante el proceso y su influencia en los resultados posteriores. Esta percepción de control ha sido repetidamente referida en la literatura como una influencia primordial en las reacciones de los adultos ante sus situaciones vitales (Heckhausen y Schultz, 1995). Esto parece particularmente relevante en los contextos laborales que son vistos como dominios en los que los trabajadores ejercen control personal sobre sus procesos y sus resultados (Barnes-Farrell, 2003). En el caso de los directivos entrevistados se pueden distinguir dos situaciones diferentes en términos de voluntariedad de la decisión de acogerse al plan de prejubilación. Por una parte están los directivos que, incluso más que aceptar voluntariamente la propuesta de la organización, son ellos los que llevan toda la iniciativa y luchan por acceder al programa, y por otra parte están los otros directivos que muestran un caso de voluntariedad forzosa o de aceptación involuntaria, con ausencia total de control sobre la situación. Asociado directamente a estas dos diferentes formas de control se puede vincular las evidencias sobre las consecuencias del proceso para la persona. Los procesos de prejubilación de los cuatro directivos con experiencias satisfactorias fueron controlados y preparados directamente por nuestros entrevistados.

En consecuencia, este trabajo también nos permite afirmar que en el cierre adelantado del ciclo profesional por cuenta ajena pueden existir procesos plenamente satisfactorios. Pero, mientras los directivos que controlaron sus procesos manifiestan experiencias positivas de su nueva situación, los directivos que no lo controlaron, o bien narraron vivencias contradic- 
torias e inconsistentes o estas fueron claramente negativas. En este segundo grupo, en concreto, se puede sugerir que se pone de manifiesto más intensamente la importancia de las estrategias de afrontamiento ante la salida inesperada de la organización. Los casos de la experiencia de voluntariedad forzosa o de aceptación involuntaria, producen una primera reacción de duelo o de bloqueo que parece que luego debe ser superada con una reorganización de la vida post-laboral mediante el afrontamiento de la nueva situación. Este hecho, como decíamos antes, parece coherente con cierta literatura reciente (Wang, 2007).

Ahora bien, si como se ha señalado antes (Barnes-Farrell, 2003), los contextos laborales son dominios en los que el diseño del puesto, la recompensa económica y el ambiente organizacional han cimentado la creencia del trabajador sobre su control de los acontecimientos vitales, esta creencia es probable que se halle mucho más asentada entre los directivos. En este sentido, nuestros datos parecen sugerir que para los ejecutivos entrevistados la decisión sobre su acceso a la prejubilación es percibida como un fenómeno personal en el que no desea que se interfiera. En consecuencia, se trata de una experiencia personal que todos abordaron con independencia de sus entornos familiares más próximos y de sus redes sociales extra-familiares. Además de la decisión, los directivos manifiestan que asumen las vivencias del proceso y las consecuencias personales y psicosociales con toda responsabilidad. No muestran actitudes victimistas, sino que los directivos se responsabilizan de la situación y asumen que son ellos los que deben salir adelante, que son ellos los que diseñan y gestionan su destino. Tanto si su situación personal es positiva como si es negativa, no eluden el protagonismo de sus propias vidas. En ningún momento realizan atribuciones externas, que podrían ser protectoras de su bienestar psicológico sino que los directivos parece que realizan otra interpretación de la experiencia de la prejubilación. Asumen la soberanía sobre sus vidas, unas veces con disciplina, otras con entusiasmo, pero siempre son ellos los titulares de sus carreras personales.

Curiosamente, en contra de lo que propone la literatura (Barnes-Farrell, 2003), en los participantes de este estudio no se ve que la percepción de pérdida de control personal dispare los llamados mecanismos secundarios, tales como una revalorización del retiro. Es llamativo, por ejemplo, que en las entrevistas ningún directivo mencionó la salud como un elemento que precipitase la decisión de prejubilarse, especialmente tratándose de un grupo de personas todas ellas mayores de 55 años, entre quienes podría esperarse algún pequeño deterioro de la salud. Para quienes el control se perdió, la pregunta al día siguiente fue: “¿Qué hice mal para estar en esta situación?"

Por último, en este trabajo de investigación se podría plantear si el colectivo de personas perteneciente a la alta dirección de las organizaciones forman un "perfil de prejubilados" por la condición derivada de su cargo en la organización. De hecho, las revisiones teóricas reclaman por la inexistencia de investigación empírica que explore los intrincados lazos entre el prestigio ocupacional o el estatus socioeconómico previo y el bienestar posterior (Szinovacz, 2003), señalando además que los pocos estudios existentes aportan resultados contradictorios (Calansanti, 1988; Dorfman, Kohout y Heckert, 1985; Matthews y Brown, 1987; Richardson y Kilty, 
1991; Szinovacz, 1989). Sin embargo, a la vista del contenido de los testimonios de los ejecutivos y del exiguo tamaño de la muestra utilizada, aquí tampoco puede concluirse que los prejubilados de la alta dirección presenten reacciones específicas exclusivas derivadas de su estatus profesional, aunque sí es cierto que tienen algunas características compartidas entre ellos y que podrían ser diferentes de las que manifiesta el personal de base.

A diferencia de los discursos de algunos de los miembros de base prejubilados, recogidos en la investigación precedente (Alcover y Crego, 2005; Crego y Alcover, 2008; Crego et al., 2008), los ejecutivos de este trabajo no asimilan la prejubilación a falsos periodos vacacionales, ni manifiestan euforia por la ruptura de una relación laboral traumática previa. En muchos de ellos no aparece ninguna manifestación de rechazo hacia la organización. No se incluyen valoraciones cualitativas del acontecimiento. Estos directivos no expresan incomprensión. Su discurso es meramente descriptivo. La mayor implicación e identificación de los directivos con las estrategias y objetivos de la organización podría ser la explicación de esta comprensión y aceptación que manifiestan estos entrevistados. Estos factores vinculados al contenido de la tarea, junto con la capacidad de desarrollar alguna actividad posterior a la salida y unido a la cobertura segura de sus necesidades económicas podrían estar condicionando la positiva valoración de la propuesta de prejubilación.

En resumen, y a pesar de lo reducido de la muestra de este trabajo exploratorio, se han observado cuatro experiencias plenamente satisfactorias, una experiencia altamente aversiva, y dos relatos con secuencias del discurso contradictorias. Tanto la experiencia aversiva como las dos inconsistentes han sido evidenciadas en los procesos de prejubilación en los que el control estuvo en poder de la organización. Una economía familiar desahogada y asegurada, una amplia red social, perfiles competitivos y ambiciosos, una mayor experiencia en la toma de decisiones o en la resolución de problemas, no garantizan la inmunización contra los riesgos psicosociales y psicológicos que puede provocar una inadecuada gestión del proceso de la prejubilación. Por el contrario, y por el lado de las desventajas, la identificación con la organización, la total implicación con los proyectos de la empresa, su mayor estatus social y económico, son aspectos, entre otros, que pueden influir de forma más negativa sobre la identidad personal, la autoestima y el autoconcepto de los directivos que del resto de trabajadores, haciendo que el ajuste a la condición de prejubilados sea difícil para ellos.

\section{Limitaciones del presente estudio y sugerencias para futuras investigaciones}

Este estudio adolece de importantes limitaciones, entre las que la más destacada es la limitación de la muestra. Pese a las enormes dificultades que implica el acceso a estos colectivos y a sus discursos relativos a experiencias con alto contenido emocional, es necesario abordar en estudios posteriores muestras más amplias y con diversidad de género.

Otra limitación procede del diferente formato de recogida de la información entre las entrevistas grabadas y aquellas en que sólo se autorizó la transcripción 
textual. En el presente estudio se han tratado por igual notas y grabaciones, no obstante, esto puede suponer una limitación ya que se han agregado datos recogidos con procedimientos diversos. Desde la perspectiva del análisis cualitativo, también hay que incorporar en el estudio de los discursos la fuerza comunicativa del lenguaje no verbal. Sin ninguna duda, todas las conclusiones que se alcanzan en este informe están soportadas por la comunicación no verbal, tanto vocal como no vocal, pero este elemento no se ha podido consignar en el análisis.

En resumen, es necesario ampliar las investigaciones sobre la aversividad de la experiencia de la prejubilación con el objeto de concienciar a las empresas de lo "traumático" que puede llegar a ser para las personas este procedimiento, que por el contrario resulta "no traumático" para las organizaciones. En este sentido, la posibilidad de diseñar programas de prevención en los planes de prejubilación contribuirá a minimizar los efectos de la experiencia. Las experiencias específicas de los altos directivos pueden brindar algunas claves sobre las que continuar investigando, ya que el control sobre el proceso y la preparación de estrategias de afrontamiento eficientes son alguna de las herramientas que permitirán afrontar la prejubilación como una oportunidad en lugar de cómo una amenaza.

En este análisis ha vuelto a ponerse de manifiesto que la prejubilación es un fenómeno tan diverso y complejo como lo son las personas que pasan por él. Su simplificación, a través de un tratamiento exclusivamente economicista, representa una prueba más de la deshumanización de la vida económica de las sociedades desarrolladas actuales. Por el contrario, se reco- mienda ampliar el marco de las investigaciones con el objetivo final de conciliar los intereses de las organizaciones con el desarrollo pleno y satisfactorio de los profesionales, "de las personas", que alcanzan determinadas edades desempeñando alguna función dentro de una organización.

\section{REFERENCIAS}

Aguilar, J. M. (1999). Entrevista en profundidad. Buenos Aires: Ed. Lumen Humanitas.

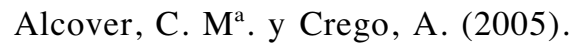
Factores implicados en la decisión de retiro laboral temprano: aproximaciones desde el análisis del discurso de una muestra de prejubilados españoles. Revista de Psicología Social Aplicada, 15, 133-163.

Alcover, C. M ${ }^{\mathrm{a}}$, Crego, A., Guglielmi, D., y Chiesa, R. (2009). Psychosocial profiles for the perceived outcomes of early work retirement: a comparison of the Spanish (involuntary) and Italian (voluntary) EWR models (manuscrito sometido a proceso de revisión).

Atchley, R. (1989). A continuity theory of normal aging. The Gerontologist, 29, 183-190.

Barnes-Farrell, J. (2003). Beyond Health and Wealth: attitudinal and other influences on retirement decision-making. En G. Adams y T. Beehr (Eds.), Retirement: reasons, processes and results (pp. 159-187). New York: Springer.

Calasanti, T. M. (1988). Participation in dual economy and adjustment to retirement. International. Journal of Aging and Human Development, 26, 13-27. 
La prejubilación en los miembros de alta dirección de las organizaciones: un análisis cualitativo

Corbetta, P. (2007). La entrevista cualitativa. En: P. Corbetta (Ed.). Metodología y técnicas de investigación social. ( $p p$. 343-374). Madrid: McGraw Hill.

Crego, A. y Alcover, C. $\mathrm{M}^{\mathrm{a}}$. (2008). Vidas reinterpretadas. Una aproximación psicosocial a las experiencias de prejubilación. Madrid: Dykinson - Servicio de Publicaciones de la Universidad Rey Juan Carlos.

Crego, A., Alcover, C. Mª y MartínezÍñigo, D. (2008). The transition process to post-working life and its psychosocial outcomes: A systematic analysis of Spanish early retirees' discourse. Career Development International, 13, 186-204.

Davis, M. (2003). Factors related to bridge employment participation among private sector early retirees. Journal of Vocational Behavior, 63, 55-71.

Denis, J-L., Lamothe, L. y Langley, A. (2001). The dynamics of collective leadership and strategic change in pluralistic organizations. Academy of Management Journal, 44, 809-37.

Dorfman, L. T., Kohout, F. J., y Heckert, D. A. (1985). Retirement satisfaction in the rural elderly. Research on Aging, 7, 577-599.

Feldman, D. (1994). The decision to retire early. Academy of Management Review, 19, 285-311.

Feldman, D. (2003). Endgame: The Design and Implementation of Early Retirement incentive programs. En G. Adams y T. Beehr (Eds.), Retirement: reasons, processes and results (pp. 83-114). New York: Springer.
Feldman, D., y Kim, S. (2003). Bridge employment during retirement: a field study of individual and organizational experiences with post-retirement employment. Human Resource Planning, 23, 1425 .

Freedman, V. A., Martin, L., y Schoeni, R. (2002). Recent trends in Disability and Functioning Among Older Americans: A critical Review of the Evidence. Journal of the American Medical Association, 288.

Hambrick, D. C. y Mason, P. A. (1984). Upper echelons: the organization as a reflection of its top managers. Academy of Management Review, 9, 193-206.

Hambrick, D. C., Cho, T. S. y Chen, M-J. (1996). The influence of top team management heterogeneity on firms' competitive moves. Administrative Science Quarterly, 41, 659-84.

Heckhausen, J., y Schultz, R. (1995). A life-span theory of control. Psychological Review, 102, 284-304.

Hobfoll, S. (2002). Social and psychological resources and adaptation. Review of General Psychology, 6, 307-324.

Kim, S., y Feldman, D. (2000). Working in retirement: the antecedents of bridge employment and its consequences for quality of life in retirement. Academy of Management Journal, 43, 1191- 1210.

López Cumbre, L. (1991). El sistema de prejubilación en España: una experiencia de muchos años. Revista de Trabajo y Seguridad Social, 1, 47-82

López Cumbre, L. (2001). Prestaciones complementarias de prejubilación: un 
debate sobre su naturaleza retributiva o de protección social. Comentario a la Sentencia del TJCE de 13 de julio de 2000, Caso Defreyn/Sabena, SA. Revista del Ministerio de Trabajo y Asuntos Sociales, 32, 183-199.

López Cumbre, L. (1998). La prejubilación. Madrid: Civitas/ UAM.

Lund, Th., y Villadsen, E. (2005). Who retires early and why? Determinants of early retirement pension among Danish employees 57-62 years. European Journal of Ageing, 2, 275-280.

Madrid, A. J., y Garces de los Fayos, E. (2000). La preparación para la jubilación: Revisión de los factores psicológicos y sociales que inciden en un mejor ajuste emocional al final del desempeño laboral Anales de Psicología, 16, 87-99.

Martinez, P. C., Lozano, J. A. F., Ancizu, I., Valdés, C. A., y Candenas, J. A. (2003). Repercusiones psicológicas y sociales de la prejubilación. [Psychological and sociological repercussions of early retirement]. Psicothema, 15, 49-53.

Matthews, A. y Brown, K. (1987). Retirement as a critical life event. The differential experiences of men and women. Research on aging, 9, 548-571.

McArdle, A., Vasilaki, A., y Jackson, M. (2002). Exercise and skeletal muscle aging: Cellular and molecular mechanisms. Aging Research Reviews, 1, 79-93.

Navarro, P. y Díaz, C. (1999). Análisis de contenido. En: J. M. Delgado y J. Gutiérrez (Coords.). Métodos y técnicas cualitativas de investigación en ciencias sociales. (pp. 177-224). Madrid: Síntesis.
Park, D. C. (2000). The basic mechanisms accounting for age-related decline in cognitive function. En D. C. Park y N. Schwarz (Eds.), Cognitive aging: $A$ primer (pp. 3-22). Philadelphia: Psychology Press.

Peiro, J. y Prieto, F. (1996). Tratado de psicología del trabajo. Vol. 1. Madrid: Síntesis.

Pinquart, M. y Schindler, I. (2007). Changes of Life satisfaction in the transition to retirement: A latent class approach. Psychology and Ageing, 22, 442-455.

Pitcher, P. y Smith, A. D. (2001). Top management heterogeneity: personality, power, and proxies. Organization Science, $12,1-18$.

Richardson, V. y Kilty, K. (1991). Gender differences in mental health before and after retirement: A longitudinal analysis. Journal of Women and Aging, 7, 19-36.

Schmitt, N., Coyle, B. W., Rauschenberger, J. y White, J. K. (1979). Comparison of early retirees and nonretirees. Personnel Psychology, 32, 327-340.

Schoeni, R., Freedman, V. A., y Martin, A. (2005). Socioeconomic and Demographic Disparities in Trends in Old - Age disability. Trends. Working Paper, 5, 1-26.

Shultz, K. S., Morton, K. R., y Weckerle, J. R. (1998). The influence of push and pull factors on voluntary and involuntary early retirees' retirement decision and adjustment. Journal of Vocational Behavior, 53, 45-57.

Szinovacz, M. (1989). Decision-Making on Retirement Timing. En D. Brinberg 
La prejubilación en los miembros de alta dirección de las organizaciones: un análisis cualitativo

y J. Jaccard. (Eds.), Dyadic Decision Making. New York: Springer.

Szinovacz, M. (2003). Contexts and Pathways: retirement as an institution, process and experience. En G. Adams y T. Beehr (Eds.), Retirement: reasons, processes and results (pp.6-52). New York: Springer.

Taylor, M. y Doverspike, D. (2003). Retirement planning and preparation. En G. Adams y T. Beehr (Eds.), Retirement: reasons, processes and results (pp. 5382). New York: Springer.

Topa, G., Moriano, J, Depolo, M., y
Morales, J. F. (2009). Bridge employment and retirees' personal wellbeing. A structural equation model with a European probabilistic sample. Psicothema, 21, 285292.

Vallés, M. (1997). Técnicas cualitativas de investigación social. Reflexión metodológica y práctica profesional. Madrid: Síntesis.

Wang, M. (2007). Profiling retirees in the retirement transition and adjustment process: examining the Longitudinal Change Patterns of retirees' psychological well-being. Journal of Applied Psychology, 92, 455-474. 